Pacific Journal of Mathematics

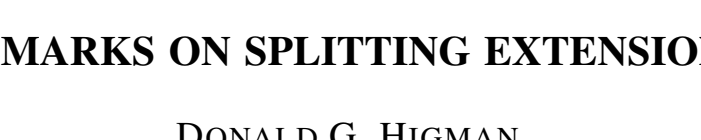




\title{
REMARKS ON SPLITTING EXTENSIONS
}

\author{
D.G. HIGMAN
}

1. Introduction. If $N$ is a normal subgroup of the finite group $G$ we call $G$ an extension of $N$. Such an extension $G$ over $N$ is said to split if there exists a complement of $N$ in $G$, that is, if there exists a subgroup of $G$ which contains exactly one element from each coset of $G$ modulo $N$. A frequently used criterion for splitting is provided by a theorem of Schur, namely, if $N$ has order prime to its index in $G$, then $G$ splits over $N$. W. Gaschütz [1] has recently given a generalization of this theorem for the case when $N=A$ is abelian, which states that (i) $G$ splits over $A$ if and only if there is for each prime $p$ a p-Sylow subgroup $S$ of $G$ which splits over $S \cap A,{ }^{1}$ and (ii) there exists a subgroup $U<G$ such that $G=A U$ if and only if there exists for some prime p a p-Sylow subgroup $S$ of $G$ and a subgroup $V$ of $S$ such that

$$
S=[S \cap A] V \text {, and } \eta_{G}(V \cap A)=S \cap A .^{1}
$$

llere $n_{G}(V \cap A)$ denotes the subgroup generated by all the conjugates to $V \cap A$ in $G$.

In $\& 2$ of this note we apply part (i) of the theorem of Gaschütz to establish a generalization of the theorem of Schur for non-abelian extension. In $\$ 3$ we apply (ii) to obtain a characterization of extensions $G$ over $N$ such that $N$ is contained in the Frattini subgroup. The remaining two sections are concerned with the question of conjugacy of complements.

Nota TIONS. Group will always mean finite group unless the contrary is explicitly stated. For $H$ a subgroup of a group $G,[G: h]=\operatorname{index}$ of $H_{i}$ in $G$. For $Y$ a set of elements of $G,\{Y\}=$ subgroup generated by the elements of $Y$. If $A$ and $B$ are groups, $A \times B$ denotes their direct product. $A \leq B$ means $A$ is con-

${ }^{1}$ Since any two $p$-Sylow subgroups of $G$ are conjugate, this condition is satisfied by all $p$-Sylow subgroups whenever it is satisfied by any one of them. The condition is automatically satisfied by those $p$-Sylow subgroups of $G$ for which $p$ does not divide both the order and the index of the normal subgroup.

Received July 8, 1952.

Pacific J. Math. 4 (1954), $545-555$ 
tained in $B$, while $A<B$ means proper inclusion. $A \cap B=$ set theoretic intersection of $A$ and $B$.

2. A subgroup $C$ of $G$ is a complement for the extension $G$ over $N$ if and only if $G=N C$ and $1=N \cap C$.

THEOREM 1. A subgroup $C$ of a group $G$ is a complement for the extension $G$ over $N$ if and only if $C$ is minimal with respect to the property $G=N C$, and there exists for each prime $p$ a p-Sylow subgroup $S$ of $G$, and a complement of $N \cap S$ in $S$ which is part of $C .^{1}$

Proof. Assume that $C$ is a complement of $N$ in $G$. Then clearly $C$ is minimal with respect to the property $G=N C$. If $P$ is a p-Sylow subgroup of $C$, and if $S$ is a $p$-Sylow subgroup of $G$ such that $P \leq S$, then $P$ is a complement of $S \cap N$ in $S$. For, since $P \leq C, N \cap P \leq N \cap C=1$. And since $P \leq S,[S \cap N] P \leq S$. But $S \cap N$ is a $p$-Sylow subgroup of $N$, and $P$ is a p-Sylow subgroup of $C$, from which it follows that $[S \cap N] P$ is a $p$-Sylow subgroup of $G$. Hence $[S \cap N] P=S$. We have proved the necessity of the condition of the theorem.

Now assume conversely that this condition is satisfied. Let $P$ be a Sylow subgroup of $M=N \cap C, x$ an element of $C$. Since $M$ is a normal subgroup of $C$, $P^{x}$ is also a Sylow subgroup of $M$ for the same prime. Hence there is an element $y$ in $M$ such that $P^{x y}=P$. Then $x y$ is in the normalizer $T$ of $P$ in $C$, that is $x$ is in $M T$. Hence $C=M T$, so that $G=N C=N M T=N T$. Hence by the minimality property of $C, T=C$. We have shown that each Sylow subgroup of $M$ is normal in $C$, that is, that $M$ is nilpotent. ${ }^{2}$ We must prove that $M=1$.

If $p$ is a prime, there exists by our assumption a p-Sylow subgroup $S$ of $G$, and a complement $U$ of $S \cap N$ in $S$ which is part of $C$. Since $U \leq S, U$ is a psubgroup of $C$. If $Q$ is a $p$-Sylow subgroup of $C$ such that $U \leq Q$, then $U$ is a complement of $M \cap Q$ in $Q$. For, let $P$ be a $p$-Sylow subgroup of $G$ such that $Q \leq P$. Then there is an element $x$ in $G$ such that

$$
P=S^{x}=[S \cap N]^{x} U^{x}=[P \cap N] U^{x} .
$$

Hence, since $1=U \cap N, P=[P \cap N] U$, so that

$$
Q=Q \cap P=[Q \cap N] U=[Q \cap M] U
$$

2 The condition that $C$ be minimal with respect to the property $G=N C$ is equivalent to the condition $M=N \cap C \leq \phi(C)$. We may infer the nilpotency of $M$ from the nilpotency of $\phi(C)$ (c.f. $\{3$ ). 
For $X$ a subgroup of $G$, set $\bar{X}=M^{\prime} X / M^{\prime}$, where $M^{\prime}$ denotes the commutator subgroup of $M$. Then $\bar{Q}$ is a $p$-Sylow subgroup of $\bar{C}$, and since $M^{\prime} U \cap M=M^{\prime}[U n$ $M]=M^{\prime}, \bar{U}$ is a complement of $\bar{Q} \cap \bar{M}$ in $C$. Hence, since $\bar{M}$ is abelian, there exists by part (i) of the theorem of Gaschütz a complement $\bar{D}=D / M^{\prime}$ of $\bar{M}$ in $\bar{C}$. But then $C=M D$ and $M^{\prime}=M \cap D$. Since $M$ is nilpotent, $M \neq 1$ implies $M \cap D=$ $M^{\prime}<M=M \cap C$, that is $D<C$. Since $G=N C=N M D=N D$, this contradicts the minimality property of $C$. Hence $M=1$, which proves the sufficiency of the condition.

Corollary (Schur's theorem). If $N$ has order prime to its index in $G$, then $G$ splits over N.

REMARK. Theorem 1 does not, of course, settle the question of the necessity of the hypothesis that $N$ be abelian for the theorem of Gaschütz. ${ }^{3}$

The following example shows that in a splitting extension $G$ over $N$, not every subgroup $C$ which is minimal with respect to the property $G=N C$ need be a complement, even when $N$ is abelian.

EXAMPLE. Let $M \neq 1$ be an abelian normal subgroup of the group $C$, and assume that $M$ is contained in the Frattini subgroup $\phi(C)$ of $C(c . f . \S 3)$. Since $\phi(C)$ is nilpotent it will have a center $\neq 1$; we may take, for instance, $M=$ the center of $\phi(C)$. By a theorem of Artin [2, p. 103] there exists a free abelian group $A$ of finite rank, and an (infinite) group $G$ such that if we set $N=M \times A$, then

1. $G$ is a splitting extension of $N$.

2. $G=N C$

3. $M=N \cap C$.

By the choice of $M$ and $C$, no proper subgroup of $C$ satisfies 2.

Let now $m$ be the order of $M$. Since $N$ is abelian, $N^{m}$ [ $=$ the totality of $m$ th powers of elements of $N]$ is a characteristic subgroup of $N$, and hence is normal in $G$. Furthermore, $N^{m} \cap M=1$. Since $N$ is abelian of finite rank, and since $G / N$ is finite, as an isomorphic image of the finite group $C / M, G / N^{m}$ is finite. Set $\bar{G}=G / N^{m}, \bar{N}=N / N^{m}$ and $\bar{C}=N^{m} C / N^{m}$. Since the extension $G$ over $N$ splits, so does $\bar{G}$ over $\bar{N} . \bar{C}$ is minimal with respect to the property $\bar{G}=\bar{N} \bar{C}$, but

$$
\bar{C} \cap \bar{N}=M N^{m} / N^{m} \simeq M \neq 1 \text {. }
$$

THEOREM 2. For an extension $G$ over $N$ the following five conditions are

${ }^{3}$ That this hypothesis actually is necessary has been shown by Professor Zassenhaus. See the note at the end of this paper. 
equivalent.

(1) N has order prime to its index in $G$.

(2) if $H$ is a subgroup of $G$, then

(a) there exists a complement of $N \cap H$ in $H$.

(b) if either $H /[N \cap H]$ or $N \cap H$ is solvable, then any two complements of $N \cap H$ in $H$ are conjugate in $H$.

(3) for each prime divisor $p$ of the order of $N$, there exists a p-Sylow subgroup $S$ of $N$ such that if $T$ denotes the normalizer of $S$ in $G$,

(a) there exists a complement of $N \cap T$ in $T$.

(b) if $H$ is a nilpotent subgroup of $T$, then any two complements of $N \cap H$ in $H$ are conjugate in $H$.

(4) if $H$ is a nilpotent subgroup of $G$, then

(a) there exists a complement of $N \cap H$ in $H$.

(b) any two complements of $N \cap H$ in $H$ are conjugate in $H$.

(5) if $H$ is a nilpotent subgroup of $G$, then there exists a subgroup $C$ of $H$ such that for each subgroup $U$ of $H, U=[U \cap N] \times[U \cap C]$.

Proof. Assume that $N$ has order prime to its index in $G$, then clearly the same is true of the normal subgroup $N \cap H$ of $H$, for any subgroup $H$ of $G$. Hence $H$ splits over $N \cap H$ by the theorem of Schur. Furthermore, by a theorem of Zassenhaus [2, p. 132] if either $H /[N \cap H]$ or $N \cap H$ is solvable then any two complements for this extension are conjugate in $H$. Thus $(2)$ is a consequence of (1).

Conditions (3) and (4) are immediate consequences of (2).

Next we shall prove that (3) and (4) each imply (1). Assume that the extension $G$ over $N$ satisfies (3), and assume that $p$ is a prime which divides both the order and the index of $N$. Then by (3), (a) there exists a p-Sylow subgroup $S$ of $N$, and a subgroup $C$ such that if $T$ denotes the normalizer of $S$ in $G, C$ is a complement of $N \cap T$ in $T$. But $G=N T$, so that $C$ is a complement of $N$ in $G$. Thus $[C: 1]=[G: N]$, hence since $p$ divides $[G: N]$, there exists an element $x$ in $C$ of order $p$. Since $x$ is in $T$, and since $p$ divides the order of $N$, there exists an element $z$ of order $p$ in $S \cap N$ such that $x z=z x$. Since $x$ is not in $N, H=\{x, z\}=\{x\} \times\{z\}$ and $N \cap H=\{z\}$, whereby it follows from (3), (b), that $\{x\}$ and $\{x z\}$ are conjugate in H. Since this is impossible, (3) implies (1).

Now assume (4), and suppose again that $p$ is a prime which divides both the order and the index of $N$. If $S$ is a $p$-Sylow subgroup of $G$, there exists by 
(4), (a), a complement $C$ of $S \cap N$ in $S$. Since $p$ divides $[G: N]$, there exists an element $x$ in $C$ of order $p$. Since $p$ divides the order of $N, S \cap N$ is a non-trivial normal subgroup of $S$. Now a repetition of the construction of the preceding paragraph leads to a contradiction with (4), ( b), proving that (4) implies (1). We have proved the equivalence of the first four conditions.

If $H$ is a nilpotent subgroup of $G,(2)$ implies the existence of a complement $C$ of $N \cap H$ in $H$, and (1) implies that the orders of $N \cap H$ and $C$ are relatively prime. Now (5) is a consequence of a property of nilpotent groups. Thus (5) is implied by the equivalent conditions (1) and (2). Conversely, if $S$ is a $p$ Sylow subgroup of $G$, (5) implies the existence of a subgroup $C$ of $S$ such that $U=[U \cap N] \times[U \cap C]$ for each subgroup $U$ of $S$. But it is well known that this implies that $S \cap N$ and $C$ have relatively prime orders. Hence one of $S \cap N$ and $C$ is trivial. This proves that (5) implies (1), completing the proof of Theorem 2 .

3. The Frattini subgroup $\phi(G)$ of the group $G$ is the intersection of $G$ with all its maximal subgroups. In this section we shall note a characterization of those normal subgroups $N$ of $G$ which are contained in $\phi(G)$. It is well known that

(a) $N \leq \phi(G)$ if and only if $G=N C, G$ a subgroup of $G$ implies $G=C$. Hence part (ii) of the theorem of Gaschütz has an equivalent statement

(b) the abelian normal subgroup $A$ of $G$ is contained in $\phi(G)$ if and only if for each prime $p$ there is a p-Sylow subgroup $S$ of $G$ such that $S=[S \cap A] V, V$ a subgroup, implies $S \cap A=\eta_{G}(V \cap A){ }^{1}$

Using (a ) it is easy to verify that

(c) if $M$ is a normal subgroup of $G$ such that $M \leq N$, then $N \leq \phi(G)$ if and only if $M \leq \phi(G)$ and $N / M \leq \phi(G / M)$.

Since $\phi(G)$ is nilpotent [2, p. 122; this can be proved using (a) together with the first part of the argument of the sufficiency proof of Theorem 1] it will suffice for the purposes of determining the normal subgroups $N$ which are contained in $\phi(G)$ to consider the case in which $N$ has prime power order.

$N^{(i)}$ denotes the $i$ th derived subgroup of $N, N^{(0)}=N, N^{(1)}=N^{\prime}$. For $X$ a subgroup of $G, n_{G}(X)$ denotes the subgroup generated by all the conjugates to $X$ in $G$.

THEOREM 3. Let $N$ be a normal subgroup of the group $G$, and assume that $N$ has p-power order, $p$ a prime. Then $N \leq \phi(G)$ if and only if there exists a 
p-Sylow subgroup $S$ of $G$ such that for all $i \geq 0, S=N^{(i)} V, V$ a subgroup, implies

$$
N^{(i)}=N^{(i+1)} \eta_{G}\left(V \cap N^{(i)}\right) \cdot{ }^{1}
$$

Proof. Assume first that $N \leq \phi(G)$. For $X$ a subgroup of $G$, write $\bar{X}=$ $N^{(i+1)} X / N^{(i+1)}$. Then $\overline{N^{(i)}}$ is an abelian normal subgroup of $G$ with $p$-power order. Furthermore, by $(\mathrm{c}), \overline{N^{(i)}} \leq \phi(\bar{G})$. Let $\bar{\zeta}$ be the $p$-Sylow subgroup of $\bar{G}$ whose existence is inferred by (b) (indeed, any $p$-Sylow subgroup will do ${ }^{1}$ ). Then $S=S / N^{(i+1)}, S$ a $p$-Sylow subgroup of $G$. If $S=N^{(i)} V, V$ a subgroup, then $S=\overline{N^{(i)}} \bar{V}$. Hence by (b) it follows that $\overline{N^{(i)}}=\eta_{G}\left(\bar{V} \cap \overline{N^{(i)}}\right)$. But

$$
\overline{N^{(i)}} \cap \bar{V}=N^{(i)} / N^{(i+1)} \cap N^{(i+1)} V / N^{(i+1)}=N^{(i+1)}\left[N^{(i)} \cap V\right] / N^{(i+1)},
$$

from which it is, easily verified that

$$
\eta_{\bar{G}}\left(\bar{V} \cap N^{(i)}\right)=N^{(i+1)} \eta_{G}\left(V \cap N^{(i)}\right) / N^{(i+1)} .
$$

Hence $N^{(i)}=N^{(i+1)} \eta_{G}\left(V \cap N^{(i)}\right)$. We have proved the necessity of the condition of the theorem.

Assume conversely that this condition is satisfied. We prove $N \leq \phi(G)$ by induction on the order of $N$. If $N=1$ there is nothing to prove. Otherwise, since $N$ is a p-group, $N^{\prime}<N$, and since the condition of the theorem is clearly satisfied by $N^{*}$ whenever it is satisfied by $N$, it follows from the induction hypothesis that $N^{\prime} \leq \phi(G) . \bar{S}=S / N^{\prime}$ is a $p$-Sylow subgroup of $\bar{G}=G / N^{\prime}$. If $\bar{V}=V / N^{\prime}$ is a subgroup of $\bar{G}$ such that $\bar{S}=\bar{N} \bar{V}, \bar{N}=N / N^{\prime}$, then $S=N V$. Now the condition of the theorem implies

$$
\bar{N}=N / N^{\prime}=N^{\prime} \eta_{G}(V \cap N) / N^{\prime}=\eta_{G}(V \cap N) / N^{\prime}=\eta_{\bar{G}}(\bar{V} \cap \bar{N}) .
$$

Hence, since $N$ is an abelian p-group it follows from (b) that $\bar{N} \leq \phi(\bar{G})$. Hence $N \leq \phi(G)$ by $(\mathrm{c})$.

4. In this section we assume that $N=A$ is abelian, and consider the problem of the conjugacy of complements of $A$ in $G$. A complement $C$ of $A$ in $G$ is in particular a set of representatives for $G$ over $A$; $C$ consists of exactly one element $c(X)$ from each coset $X$ in $G / A$. If $D$ is a second complement, $d(X)=$ $D \cap X$, then the function $t$ from $G / f_{1}$ to $A$ defined by $d(X)=t(X) c(X)$ satisfies

$$
1=t(Y)^{X} t(X Y)^{-1} t(X)
$$


for all $X, Y$ in $G / A$. (Since $A$ is abelian, all the elements $x$ in $X$ induce the same automorphism of $A$. We write $a^{X}=a^{x}$ for $a$ in $A$ ).

Conversely, if $t$ is any function from $G / A$ to $A$ which satisfies (1), then the totality $D$ of elements $d(X)=t(X)_{c}(X)$ for $X$ in $G / A$ is a complement of $A$ in $G$. Moreover

(2) two complements $C$ and $D$ which are related by $t$ are conjugate subgroups of $G$ if and only if there is an element $a$ in $A$ such that $t(X)=a^{1-X}$ for $X$ in $G / A^{3}$

Let $H$ be a subgroup of $G$ such that $A \leq H$, and set $m=[G: H]$.

THEOREM 4. If $m$ is prime to the order of $A$, if the function $t$ from $G / A$ to $A$ satisfies (1), and if $c$ is an element of $A$ such that $t(Y)=c^{1-Y}$ for all $Y$ in $H / A$, then there is an element $a$ in $A$ such that $t(X)=a^{1-X}$ for all $X$ in $G / A .^{4}$

Proof. The function $f$ defined by $f(X)=t(X) c^{X-1}$ satisfies (1), and has the property that $f(Y)=1$ for all $Y$ in $H / A$. Choose a system $L$ of left representatives for $C / A$ over $H / A$ so that each $X$ in $G / A$ has (uniquely) the form $X=$ $\bar{X} \underline{X}$, with $\bar{X}$ in $L, \underline{X}$ in $H / A$. By (1) we have

$$
1=f(\underline{X})^{\bar{X}} f(X)^{-1} f(\bar{X})=f(X)^{-1} f(\bar{X})
$$

that is $f(X)=f(\bar{X})$. Hence

$$
f(X)=f(Y)^{-X} f(X Y)=f(Y)^{-X} f(\overline{X Y}) .
$$

Taking the product over all $Y$ in $L$ we have

$$
f(X)^{m}=\prod_{Y \in L} f(Y)^{-X} \prod_{Y \in L} f(\overline{X Y})
$$

Since $m$ is prime to the order of $A$, the mapping $\alpha: a \rightarrow a^{m}$ is an automorphism of $A$ (which commutes with every other automorphism of $A$ ). Hence

\footnotetext{
${ }^{3}$ In terms of the cohomology theory of groups this means that the number of classes of conjugate complements of $A$ in $G$ is the order of the first cohomology group of $G / A$ by A.

${ }^{4}$ This result is a consequence of the 1-dimensional case, whereas (i) of the Gaschutz theorem is a consequence of the 2-dimensional case, of a general theorem in the cohomology theory of groups (see B. Eckmann, Cohomology groups and transfer, Ann. of Math., 58 (1953), $481-4.93$.
} 


$$
b=\left\{\prod_{Y \in L} f(Y)\right\} \alpha^{-1}
$$

is an element of $A$. As $Y$ runs through $L$, so does $\overline{X Y}$, hence, applying $\alpha^{-1}$ to (3) we have $f(X)=b^{-X} b=b^{1-X}$. Thus

$$
t(X)=f(X) c^{1-X}=b^{1-X} c^{1-X}=(b c)^{1-X}
$$

Theorem 4 is now proved with $a=b c$.

COROLLARY 1. If $m$ is prime to the order of $A$, then two complements $C$ and $D$ of $A$ in $G$ are conjugate in $G$ if and only if $C \cap H$ and $D \cap H$ are conjugate in H.

Proof. Let $t$ be the function relating $C$ and $D$. The subgroups $C \cap H$ and $D \cap H$ are complements of $A$ in $H$, and are related by the restriction of $t$ to $H / A$. If $C$ and $D$ are conjugate in $G$, then by (2) there exists an element $a$ in $A$ such that $t(X)=a^{1-X}$ for all $X$ in $G / A$, and hence in particular for $X$ in $H / A$. Hence by (2), $C \cap H$ and $D \cap H$ are conjugate in $H$.

If on the other hand $C \cap H$ and $D \cap H$ are conjugate subgroups of $H$, then it follows by (2) that there is an element $c$ in $A$ such that $t(Y)=c^{1-y}$ for all $y$ in $H / A$. Hence by Theorem 4 there exists $a$ in $A$ such that $t(X)=a^{1-X}$ for all $X$ in $G / A$. Hence by (2), $C$ and $D$ are conjugate in $G$. This proves the corollary.

By part (i) of the theorem of Gaschütz the extension $G$ over $A$ splits if and only if there is for each prime $p$ a $p$-Sylow subgroup $S$ of $G$ which splits over $S \cap A$. By Theorem 4 we have

Corollary 2. Let $G$ be a splitting extension of $A$. If for each prime $p$ there is a p-Sylow subgroup $S$ of $G$ such that any two complements of $S \cap A$ in $S$ are conjugate in $S$, then any two complements of $A$ in $G$ are conjugate in $G$.

Proof. We must prove that for each function $t$ satisfying (1) there is an element $a$ in $A$ such that $t(X)=a^{1-X}$ for all $X$ in $G / A$. Let $p_{i}$ be the prime divisors of the order of $A$ and let $A_{i}$ be the corresponding primary components of $A(i=1,2, \cdots, k)$. Then $A=A_{1} \times \cdots \times A_{k}$, and each $A_{i}$, being characteristic in $A$, is a normal subgroup of $G$. For each $X$ in $G / A, t(X)$ has (uniquely) the form

$$
t(X)=\prod_{i=1}^{k} t_{i}(X),
$$


with $t_{i}(X)$ in $A_{i}$. Define $T_{i}\left(A_{i} x\right)=t(A x)$ for $x$ in $G$, and let $S_{i}$ be a $p_{i}$-Sylow subgroup of $G$. We have assumed that $S_{i}$ may be chosen in such a way that there is an element $b_{i}$ in $A_{i}$ with

$$
T_{i}\left(A_{i} y\right)=b_{i}^{1-A_{i} y}
$$

for all $y$ in $S_{i}$ (indeed, any $p_{i}$-Sylow subgroup will do). By Theorem 4, there exists $a_{i}$ in $A$ such that

$$
T_{i}\left(A_{i} x\right)=a_{i}^{1-A_{i} x}
$$

for all $x$ in $G$. Hence

$$
t_{i}(A x)=T_{i}\left(A_{i} x\right)=a_{i}^{1-A_{i} x}=a_{i}^{1-A x},
$$

whereby

$$
t(A x)=\prod_{i=1}^{k} t_{i}(A x)=\prod_{i=1}^{k} a_{i}^{1-A x}=\left\{\prod_{i=1}^{k} a_{i}\right\}^{1-A x}
$$

for all $x$ in $G$, with $a=\prod_{i=1}^{k} \quad a_{i}$ an element of $A$.

5. It has been conjectured that if $N$ has order prime to its index in $G$, then any two complements of $N$ in $G$ are conjugate. The following theorem shows that this conjecture is equivalent to

$(+)$ if $G$ is a group, and $\Gamma$ a group of automorphisms of $G$ such that the orders of $\Gamma$ and $G$ are relatively prime, then for each prime $p$, there exists a p-Sylow subgroup of $G$ which is mapped onto itself by every automorphis $m$ in $\Gamma$. Thus the theorem of Zassenhaus [2, p. 132] suffices to prove $(+)$ in case either $G$ or $\Gamma$ is solvable.

THE ORE $\mathrm{M}$ 5. For an extension $G$ over $N$ such that $N$ has order prime to its index in $G$, the following are equivalent statements.

(a) if $C$ and $D$ are complements of $N$ in $G$, then they are conjugate in $\{C, D\}$.

(b) for each subgroup $H$ of $G$ such that $G=N H$, and for each pair $C, D$ of complements of $\mathrm{N} \cap \mathrm{H}$ in $\mathrm{H}$, there exists an automorphism a of $H$ such that $C=D^{\alpha}$. 
(c) for each subgroup $H$ of $G$ such that $G=N H$, for each complement $C$ of $N \cap H$ in $H$, and for each prime $p$, there exist a p-Sylow subgroup $S$ of $N \cap H$ such that $C$ is part of the normalizer of $S$.

Proof. Clearly (a) implies (b). Assume (b), and let $H$ be a subgroup of $G$ such that $G=N H$. Let $P$ be a $p$-Sylow subgroup of $N \cap H$, and let $T$ be the normalizer of $P$ in $H$. Then $H=[N \cap H] T$. Hence, since the order of $N \cap H$ is prime to its index in $H$, there exists by the theorem of Schur a complement $D$ of $N \cap H$ in $H$ which is part of $T$, that is, which normalizes $P$. If now $C$ is any complement of $N \cap H$ in $H$, there exists by (b) an automorphism of $H$ such that $C=D^{\alpha}$. Hence $C$ normalizes the $p$-Sylow subgroup $S=P^{\alpha}$ of $N \cap H$. Thus (b) implies (c).

Now assume (c), and let $C$ and $D$ be two complements of $N$ in $G$. Assume that if $\{U, V\}$ is a pair of complements of $N$ in $G$ such that the order of $\{U, V\}$ is less than the order of $H=\{C, D\}$, then $U$ and $V$ are conjugate in $\{U, V\}$. If $N \cap H$ is nilpotent, since we have assumed that the orders of $N$ and $G / N$ are relatively prime, it follows by the theorem of Zassenhaus that $C$ and $D$ are conjugate in $H$. Otherwise, there exists a prime $p$ such that the normalizer in $H$ of a $p$-Sylow subgroup of $N \cap H$ is a proper subgroup of $H$. By (c) there exist $p$ Sylow subgroups $P$ and $Q$ of $N \cap H$ which are normalized by $C$ and $D$ respectively. There exists an element $x$ in $H$ such that $P=Q^{x}$, and the complement $E=D^{x}$ of $N$ in $G$ normalizes $P$. Thus, if we let $T$ denote the normalizer of $P$ in $H,\{C, E\} \leq$ $T<H$. Now it follows by the induction hypothesis that there exists an element $y$ in $\{C, E\}$ such that $C=E^{y}=D^{x y}$. Since both $x$ and $y$ are in $H=\{C, D\}$, so is $x y$.

Added in proof. The very interesting fact, that the hypothesis that the extension be abelian is indeed necessary for Gaschütz's theorem (i), as stated in the introduction of the present note, is shown by the following example communicated to the author by Professor Zassenhaus:

Let $G$ be the group with generators $A_{i k}, B_{i}, C_{i}(i, k=1,2)$ and the defining relations

$$
\begin{aligned}
& A_{11}^{2}=A_{12}^{2}=\left(A_{11} A_{12}\right)^{2}=A_{21}^{2}=A_{22}^{2}=\left(A_{21} A_{22}\right)^{2}, A_{1 i} A_{2 k}=A_{2 k} A_{1 i} ; \\
& B_{i}^{3}=1, B_{i} A_{i 1} B_{i}^{-1}=A_{i 2}, B_{i} A_{i 2} B_{i}^{-1}=A_{i 1} A_{i 2}, B_{1} A_{2 k}=A_{2 k} B_{1}, B_{2} A_{1 k}=A_{1 k} B_{2}, \\
& B_{1} B_{2}=B_{2} B_{1} ; C_{i}^{2}=\left(C_{i} A_{i 1}\right)^{2}=\left(C_{i} A_{i 1} A_{i 2}\right)^{2}=A_{i 1}, C_{i} B_{i} C_{i}^{-1}=B_{i}^{-1},
\end{aligned}
$$


$C_{1} A_{2 k} C_{1}, C_{2} A_{1 k}=A_{1 k} C_{2}, C_{1} B_{2}=B_{2} C_{1}, C_{2} B_{1}=B_{1} C_{2}, C_{1} C_{2}=C_{2} C_{1} \quad(i, k=1,2)$.

The subgroup $N$ generated by the four elements $A_{i k}$ is the direct product of two quaternion groups with identified centers, thus $N$ is of order 32. The group $N$ is normal in $G$ and the subgroup $G_{1}$ of $G$ generated by $N, B_{1}$ and $B_{2}$ is normal too such that $G_{1} / N$ is abelian of type $(3,3)$. The factor group $G / G_{1}$ is of type $(2,2)$. Thus $G$ is of order 1152 .

The group $G$ does not split over its normal subgroup $N$. But the factor group $G_{1} / N$ is the 3-Sylow subgroup of the factor group $G / N$ such that $G_{1}$ splits over $N$ with the subgroup generated by $B_{1}$ and $B_{2}$ as representative subgroup. Moreover the factor group generated by $N, C_{1}$ and $C_{2}$ over $N$ is a 2-Sylow subgroup of $G / N$ such that the subgroup generated by $C_{1} A_{21}$ and $C_{2} A_{12}$ is a representative subgroup of order 4 .

\section{REFERENCES}

1. W. Gaschütz, Zur Erweiterungstheorie der endlichen Gruppen., J. Reine Angew. Math. 190 (1952), 93 - 107. 1949.

2. H. Zassenhaus, The theory of groups., Chelsea Publishing Company, New York,

UNIVERSITY OF ILLINOIS,

MCGill University, MONTREAL

MONTANA STATE UNIVERSITY 



\section{PACIFIC JOURNAL OF MATHEMATICS}

\section{EDITORS}

\author{
M.M. SCHIFFER * \\ Stanford University \\ Stanford, California \\ E. HEWITT \\ University of Washington \\ Seattle 5, Washington
}

\section{R.P. DILWORTH}

California Institute of Technology Pasadena 4, California

\section{E. F. BECKENBACH**}

University of California

Los Angeles 24, California

\section{ASSOCIATE EDITORS}

$\begin{array}{llll}\text { H. BUSEMANN } & \text { P.R. HALMOS } & \text { BØRGE JESSEN } & \text { J. J. STOKER } \\ \text { HERBERT FEDERER } & \text { HEINZ HOPF } & \text { PAUL LÉVY } & \text { E.G. STRAUS } \\ \text { MARSHALL HALL } & \text { R.D. JAMES } & \text { GEORGE PÓLYA } & \text { KÔSAKU YOSIDA }\end{array}$

\section{SPONSORS}

UNIVERSITY OF BRITISH COLUMBIA

CALIFORNIA INSTITUTE OF TECHNOLOGY

UNIVERSITY OF CALIFORNIA, BERKELEY

UNIVERSITY OF CALIFORNIA, DAVIS

UNIVERSITY OF CALIFORNIA, LOS ANGELES

UNIVERSITY OF CALIFORNIA, SANTA BARBARA

UNIVERSITY OF NEVADA

OREGON STATE COLLEGE

UNIVERSITY OF OREGON
UNIVERSITY OF SOUTHERN CALIFORNIA STANFORD RESEARCH INSTITUTE STANFORD UNIVERSITY WASHINGTON STATE COLLEGE UNIVERSITY OF WASHINGTON

AMERICAN MATHEMATICAL SOCIETY HUGHES AIRCRAFT COMPANY

Mathematical papers intended for publication in the Pacific Journal of Mathematics should be typewritten (double spaced), and the author should keep a complete copy. Manuscripts may be sent to any of the editors. Manuscripts intended for the outgoing editors should be sent to their successors. All other communications to the editors should be addressed to the managing editor, E.G. Straus, at the University of California Los Angeles 24, California.

50 reprints of each article are furnished free of charge; additional copies may be obtained at cost in multiples of 50 .

The Pacific Journal of Mathematics is published quarterly, in March, June, September, and December. The price per volume (4 numbers) is $\$ 12.00$; single issues, $\$ 3.50$; back numbers (Volumes $1,2,3$ ) are available at $\$ 2.50$ per copy. Special price to individual faculty members of supporting institutions and to individual members of the American Mathematical Society: $\$ 4.00$ per volume; single issues, $\$ 1.25$.

Subscriptions, orders for back numbers, and changes of address should be sent to the publishers, University of California Press, ,Berkeley 4, California.

Printed at Ann Arbor, Michigan. Entered as second class matter at the Post Office, Berkeley, California.

* To be succeeded in 1955, by H.L. Royden, Stanford University, Stanford, California.

** To be succeeded in 1955, by E.G. Straus, University of California, Los Angeles 24, Calif.

UNIVERSITY OF CALIFORNIA PRESS - BERKELEY AND LOS ANGELES 


\section{Pacific Journal of Mathematics}

\section{Vol. 4, No. $4 \quad$ August, 1954}

Paul Civin, Orthonormal cyclic groups .................... 481

Kenneth Lloyd Cooke, The rate of increase of real continuous solutions of

algebraic differential-difference equations of the first order ......... 483

Philip J. Davis, Linear functional equations and interpolation series . . . . . 503

F. Herzog and G. Piranian, Sets of radial continuity of analytic functions . . 533

P. C. Rosenbloom, Comments on the preceding paper by Herzog and

Piranian ............................................. 539

Donald G. Higman, Remarks on splitting extensions .............. 545

Margaret Jackson, Transformations of series of the type ${ }_{3} \Psi_{3} \ldots \ldots \ldots \ldots 557$

Herman Rubin and Patrick Colonel Suppes, Transformations of systems of relativistic particle mechanics ....................... 563

A. Seidenberg, On the dimension theory of rings. II .............. 603

Bertram Yood, Difference algebras of linear transformations on a Banach

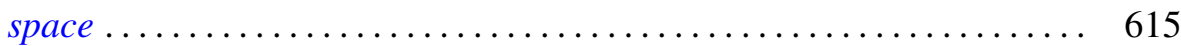

